\title{
Displacemon Electromechanics: How to Detect Quantum Interference in a Nanomechanical Resonator
}

\author{
K. E. Khosla, ${ }^{1,2}$ M. R. Vanner, ${ }^{3,4}$ N. Ares, ${ }^{5}$ and E. A. Laird ${ }^{6,5, *}$ \\ ${ }^{1}$ Center for Engineered Quantum Systems, The University of Queensland, Brisbane, \\ Queensland 4072, Australia \\ ${ }^{2}$ School of Mathematics and Physics, The University of Queensland, Brisbane, Queensland 4072, Australia \\ ${ }^{3}$ QOLS, Blackett Laboratory, Imperial College London, London SW7 2BW, United Kingdom \\ ${ }^{4}$ Clarendon Laboratory, Department of Physics, University of Oxford, Oxford OX1 3PU, United Kingdom \\ ${ }^{5}$ Department of Materials, University of Oxford, Parks Road, Oxford OX1 3PH, United Kingdom \\ ${ }^{6}$ Department of Physics, Lancaster University, Lancaster LA1 4YB, United Kingdom
}

(Received 30 October 2017; revised manuscript received 28 March 2018; published 24 May 2018)

\begin{abstract}
We introduce the "displacemon" electromechanical architecture that comprises a vibrating nanobeam, e.g., a carbon nanotube, flux coupled to a superconducting qubit. This platform can achieve strong and even ultrastrong coupling, enabling a variety of quantum protocols. We use this system to describe a protocol for generating and measuring quantum interference between trajectories of a nanomechanical resonator. The scheme uses a sequence of qubit manipulations and measurements to cool the resonator, to apply two effective diffraction gratings, and then to measure the resulting interference pattern. We demonstrate the feasibility of generating a spatially distinct quantum superposition state of motion containing more than $10^{6}$ nucleons using a vibrating nanotube acting as a junction in this new superconducting qubit configuration.
\end{abstract}

DOI: 10.1103/PhysRevX.8.021052

Subject Areas: Condensed Matter Physics,

Mesoscopics, Quantum Physics

\section{INTRODUCTION}

The superposition principle is a fundamental tenet of quantum mechanics, and it is essential to understand a wide range of quantum phenomena. As the scale of quantum objects increases, the experimental consequences of this principle become increasingly hard to isolate. Is there a scale at which this tenet begins to break down? The strongest tests of superposition come from matter-wave interferometry between trajectories of large molecules. Remarkably, interference can be measured using molecules of mass exceeding $10^{4}$ atomic mass units (amu) $[1,2]$. The ability to create unambiguous superpositions on a mesoscopic scale would allow tests of quantum collapse theories and gravitational decoherence [3-7], ultimately addressing experimentally the question of why we fail to see superpositions in everyday life [8]. This has inspired numerous challenging proposals to detect interference of larger particles [9-11] via optomechanical coupling [12], or by using levitated nanodiamonds $[13,14]$.

\footnotetext{
*e.a.laird@lancaster.ac.uk
}

Published by the American Physical Society under the terms of the Creative Commons Attribution 4.0 International license. Further distribution of this work must maintain attribution to the author(s) and the published article's title, journal citation, and DOI.
Nanomechanical resonators span this mesoscopic mass scale ranging from about $10^{6}$ to $10^{16} \mathrm{amu}$ and therefore provide an attractive route to extend the scale over which quantum effects can be observed. Recently, cooling to the ground state [15-17], as well as elements of quantum behavior such as squeezing [18-20] and coherent qubit coupling $[15,21,22]$, has become accessible with mechanical resonators of this scale. Superconducting charge or flux qubits coupled to mechanical resonators offer a path to achieving coupling strength exceeding the qubit decoherence rate, allowing the study of mechanical quantum states [23-25]. Quantum control of mechanical systems also offers a significant potential for quantum memories and for state transfer [26,27], as well as for mass and force sensing $[28,29]$. Progress towards observing mechanical superposition states has been made in both optomechanics and electromechanics, and mechanical interference fringes have recently been observed at a classical level [30]. The observation of quantum interference, however, remains outstanding and is a key goal of this paper.

Here, we introduce the "displacemon," a device that enables strong coupling between a nanomechanical resonator and a superconducting qubit. We show how to create an effective diffraction grating that leads to an interference pattern in the resonator's displacement. The scheme works by using a sequence of manipulations on the qubit to create an effective grating with a fine pitch and therefore a large momentum displacement. In molecular interference 
experiments, the diffraction grating is typically an etched membrane; however, van der Waals interactions with the slits make this hard to extend to large particles [1]. More advanced implementations use optically defined gratings; the pitch, which sets the momentum separation of the diffracted beams, is then limited by the optical wavelength [31]. In our scheme, the pitch is limited neither by an optical wavelength nor by the size of the resonator, but by the qubit-resonator coupling strength. As we will show, this allows for diffraction gratings with a pitch narrower than the ground-state wave function.

Our proposed device uses a vibrating nanobeam that is flux coupled to a superconducting qubit, through which all manipulations and measurements are performed. As a nanobeam that optimally combines high mechanical frequency, low dissipation, and the ability to couple strongly to superconducting quantum devices, we propose a suspended carbon nanotube. Previous proposals for quantum motion in nanotubes $[32,33]$ have been based on coupling to a spin qubit; however, the coherence requirement on the qubit is stringent [34]. Here, using realistic parameters derived from experiments, we show how to construct a superconducting transmon qubit that can achieve strong and coherent coupling to the mechanical motion of a carbon nanotube. Exciting recent progress in superconducting electromechanics has provided empirical evidence for quantum behavior up to a mass scale of about $10^{14}$ amu [15,35], though the observation of mechanical interference fringes from a quantum superposition state remains outstanding. Our approach here focuses on generating effective mechanical diffraction gratings to measure quantum interference fringes in a mechanical object greater than $10^{6} \mathrm{amu}$. The largest scale at which such fringes were observed is currently set by molecule interferometry experiments [2], and this proposal enables the mass scale of objects exhibiting quantum interference fringes to be extended by nearly 3 orders of magnitude.

\section{MODEL}

In general, strongly coupling a mechanical resonator to a qubit is challenging because the best qubits are engineered to be insensitive to their environment [36]. We propose a design that is robust against electrical and magnetic noise while still achieving strong mechanical coupling. We envisage a superconducting qubit of the concentric transmon design [37] in which at least one of the junctions is a vibrating nanotube (Fig. 1). Nanotube resonators offer unique advantages for studying quantum motion [38]: (i) The zero-point amplitude is typically greater than $1 \mathrm{pm}$, much larger than other mechanical resonators; (ii) the resonant frequency is sufficiently large to allow near-ground-state thermal occupation, suppressing thermal decoherence [39,40]; (iii) a nanotube can act as a Josephson junction [41-43]; and (iv) ultraclean devices offer mechanical quality factors greater than $10^{6}$, which provide long-lived states [44].

In this design [Fig. 1(a)], the two junctions form a gradiometric superconducting quantum interference device
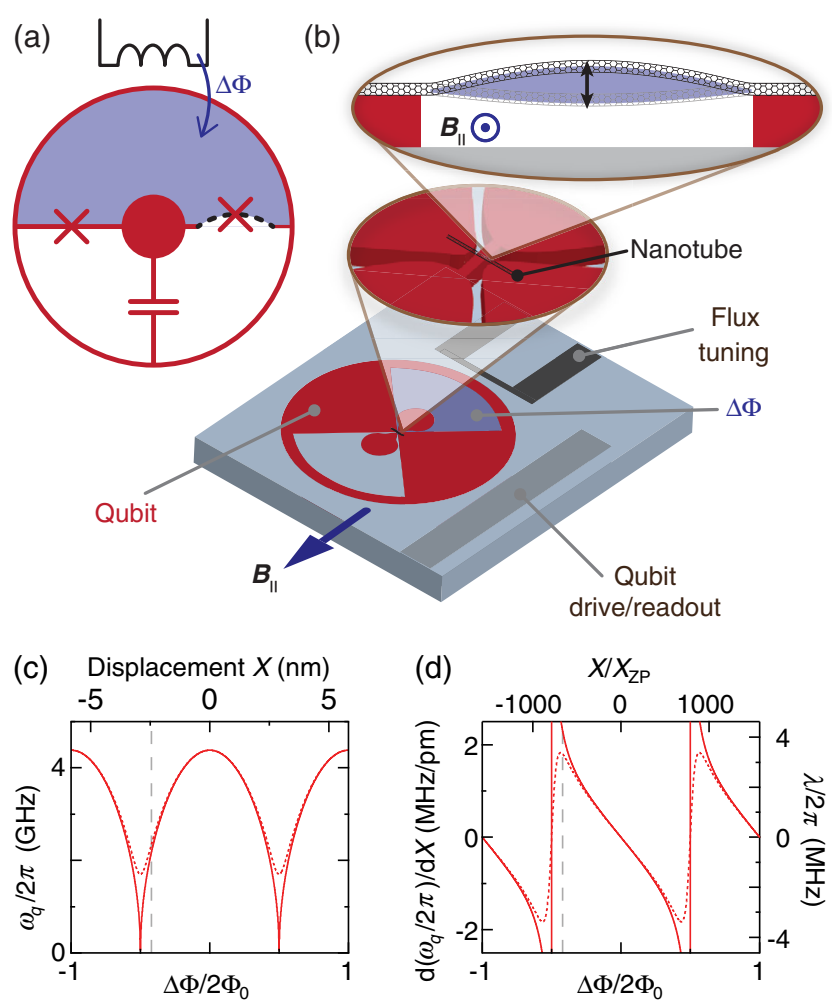

FIG. 1. Device for strong qubit-mechanical coupling. (a) Electrical schematic. The device is a gradiometric transmon qubit, biased by flux difference $\Delta \Phi$ between the SQUID loops. With a suspended nanotube acting as at least one junction (shown here for the right junction), the displacement modifies $\Delta \Phi$ and therefore modulates the qubit energy levels. (b) Arrangement of the qubit, vibrating nanotube, flux tuning coil, and drive/readout cavity antenna. The in-plane magnetic field $B_{\|}$introduces strong coupling between the vibrations and $\Delta \Phi$. (c) Qubit frequency as a function of flux difference, with parameters as in the text. Solid lines assume equal Josephson coupling in the two SQUID junctions; the dotted line assumes 30\% asymmetry (see Appendix A). Curves are plotted as a function of flux (bottom axis) and, equivalently, of displacement (top axis). (d) Qubit displacement sensitivity (left axis) and mechanical coupling rate (right axis) as a function of flux. The bias point that achieves the assumed coupling is indicated by a vertical dashed line.

(SQUID), so the qubit frequency is set by the flux difference $\Delta \Phi$ between the two loops. This flux difference is tuned primarily by means of a variable perpendicular field $\Delta B_{\perp}(t)$, while mechanical coupling is flux mediated using a static in-plane field $B_{\|}[45-47]$. This type of concentric transmon is insensitive to uniform magnetic fields, which has two advantages for this proposal: The qubit can be operated coherently away from a flux sweet spot [37], and any misaligned static flux will not perturb the energy levels. Both these facts are favorable for strong nanomechanical coupling. Because this variant of the transmon is designed for strong coupling to nanomechanical displacement, we refer to it as a displacemon. In this section, we derive the displacemon Hamiltonian and estimate the parameters for a feasible device. 


\section{A. Mechanical resonator}

We consider the nanotube as a beam of length $l$ and diameter $D$ and focus our studies on its fundamental vibrational mode $[38-40,44,48-50]$. The mechanical resonator Hamiltonian is $H_{m}=\hbar \Omega a^{\dagger} a$, where $a^{\dagger}(a)$ is the creation (annihilation) operator for the resonator. Typically, the restoring force for a clamped nanotube is dominated by the beam's tension $T$ [51], so the mechanical angular frequency is $\Omega=(\pi / l) \sqrt{(T / \mu)}$ and the zero-point amplitude is $X_{\mathrm{ZP}}=\sqrt{[\hbar /(2 m \Omega)]}=\sqrt{[\hbar /(2 \pi)]}(\mu T)^{-1 / 4}$, where $\mu=\pi \rho_{S} D$ is the mass per unit length and $\rho_{S}=$ $8 \times 10^{-7} \mathrm{~kg} \mathrm{~m}^{-2}$ is the sheet density of graphene. The displacement profile as a function of axial coordinate $Z$ is $\tilde{X}(Z)=X \sqrt{2} \sin [(\pi Z) / l]$, where $X \equiv\left(a+a^{\dagger}\right) X_{\mathrm{ZP}}$ is the displacement coordinate. This profile is normalized so that the root-mean-square displacement is equal to $X$ [51]. The flux coupling is proportional to the area swept out by the nanotube, which is equal to $\beta_{0} l X$, where $\beta_{0} \equiv$ $[1 /(l X)] \int_{0}^{l} \tilde{X}(Z) d Z=[(2 \sqrt{2}) / \pi]$ is a geometric coupling coefficient [46].

Nanotube resonators can also be fabricated without tension, so the restoring force is dominated by the beam's rigidity $[48,49]$. In this limit, the mechanical frequency is $\Omega=\left(22.4 / l^{2}\right) \sqrt{E D^{2} / 8 \mu}$ and the coupling coefficient is $\beta_{0}=0.831$, where $E \approx D \times 1.09 \times 10^{3} \mathrm{Pam}$ is the extensional rigidity.

\section{B. Qubit}

The qubit consists of a pair of superconducting electrodes coupled through the SQUID junctions. The qubit Hamiltonian is [52]

$$
H_{q}=4 E_{C}\left(\hat{n}-n_{g}\right)^{2}-E_{J} \cos \hat{\varphi},
$$

where $E_{C}$ is the charging energy, $E_{J}$ is the SQUID Josephson energy, and $\hat{n}$ and $\hat{\varphi}$ are the overall charge (expressed in Cooper pairs) and phase across the junctions, with $n_{g}$ being the offset charge. Here, we have neglected the qubit inductance, which makes a small contribution on the energy levels [37]. In the transmon limit $E_{J} \gg E_{C}$, we can approximate Eq. (1) by an effective Hamiltonian $H_{q} \approx \frac{1}{2} \hbar \omega_{q} \sigma_{z}$, where $\omega_{q}=\sqrt{8 E_{J} E_{C}} / \hbar$ is the qubit frequency and $\sigma_{z}$ is the standard Pauli matrix, acting on the qubit ground state $|-\rangle$ and the excited state $|+\rangle$. Qubit rotations, initialization, and projective measurement are now well-established techniques, using capacitive coupling to a microwave cavity in a circuit quantum electrodynamics architecture [37,53].

\section{Strong and ultrastrong coupling}

Strong and tunable coupling between the qubit and the mechanical resonator is achieved by flux coupling to the SQUID loops, which tunes the qubit Josephson energy.
Assuming equal critical current $I_{c}$ in the two junctions, this Josephson energy is

$$
E_{J}=E_{J}^{0}\left|\cos \frac{\pi \Delta \Phi}{2 \Phi_{0}}\right|,
$$

where $\Delta \Phi$ is the flux difference between the two loops, $E_{J}^{0}=I_{c} \Phi_{0} / \pi$ is the maximum Josephson energy, and $\Phi_{0}=h / 2 e$ is the flux quantum.

The flux difference can be tuned both directly, via a perpendicular magnetic field $B_{\perp}$, and via the displacement using a static in-plane field $B_{\|}$. We have

$$
\Delta \Phi=A \Delta B_{\perp}+2 \beta_{0} l B_{\|} X,
$$

where $A$ is the area of one SQUID loop. Since quite small perpendicular fields suffice to tune the qubit frequency over its full range, we envisage an on-chip coil to modulate $\Delta B_{\perp}(t)$ as a function of time $t[37,54]$. Substituting Eq. (2) into the definition of $\omega_{q}$ gives

$$
\frac{d \omega_{q}}{d X}=-\omega_{q}{ }^{0} \frac{\pi \beta_{0} l B_{\|}}{2 \Phi_{0}} \frac{\sin \pi \Delta \Phi / 2 \Phi_{0}}{\sqrt{\left|\cos \pi \Delta \Phi / 2 \Phi_{0}\right|}},
$$

where $\omega_{q}^{0}=\sqrt{8 E_{J}^{0} E_{C}} / \hbar$ is the maximal qubit frequency. The dependence of $\omega_{q}$ on $X$ gives rise to an electromechanical coupling, resulting in the Hamiltonian [23,36]

$$
H=\hbar \Omega a^{\dagger} a+\hbar \frac{\omega_{\mathrm{q}}}{2} \sigma_{z}+\hbar \lambda(t)\left(a+a^{\dagger}\right) \sigma_{z},
$$

where $\lambda(t)=\left(X_{\mathrm{ZP}} / 2\right) d \omega_{q} / d X$ [from Eq. (4)] is the qubitmechanical coupling strength, dynamically controlled through the field $\Delta B_{\perp}(t)$.

Achieving coherent interaction between the qubit and the resonator requires the quantum coherent coupling regime, where the maximum accessible coupling $\lambda_{0}$ exceeds both the mechanical rethermalization rate $\kappa_{\mathrm{th}}=k_{B} T /\left(\hbar Q_{m}\right)$ and the qubit decoherence rate $\gamma=1 / T_{2}$. Here, $Q_{m}$ is the mechanical quality factor and $T_{2}$ is the qubit coherence time. The large zero-point motion makes nanotube resonators particularly favorable for achieving this regime. Taking device parameters from simulation and experiment (Appendix A) leads to $\Omega / 2 \pi=125 \mathrm{MHz}, \omega_{q} / 2 \pi=2.19 \mathrm{GHz}$, and $\lambda_{0} / 2 \pi=4.2 \mathrm{MHz}$, with the flux dependence shown in Fig. 1. This is favorable for achieving the quantum coherent coupling regime since both $\kappa_{\mathrm{th}} / 2 \pi$ and $\gamma / 2 \pi$ are typically less than $1 \mathrm{MHz}$.

To create well-separated mechanical superpositions, a stronger condition is desirable; the qubit should precess appreciably within an interval during which the resonator can be considered stationary. This is the ultrastrong coupling regime, where $\lambda_{0}>\Omega$ [47]. It is possible that a device similar to that of Fig. 1 could access this regime (see Appendix A). However, here we instead suppose that effective ultrastrong coupling is engineered by modulating $\Delta B_{\perp}(t)$ at the mechanical frequency (see Sec. III B). In this 
modulated frame (similar to the toggled frame obtained by repeatedly flipping the qubit [55]), the resonator is effectively frozen and the required coupling condition is relaxed to $\lambda_{0}>\kappa_{\mathrm{th}}, \gamma$.

\section{GENERATING AND MEASURING MECHANICAL QUANTUM INTERFERENCE}

To realize the nanomechanical interferometer, we propose a series of operations and measurements on the qubit; this provides the necessary nonlinearity to generate mechanical superposition states. The core idea is that the state of the mechanical resonator is constrained by the qubit measurement outcome in the same way that the state of a particle is constrained by passing through a diffraction screen. By concatenating a series of qubit rotations and measurements, the resonator can be cooled, diffracted, and measured.

\section{A. Cooling the resonator}

The first step is to prepare the resonator close to its ground state. A mechanical frequency of $125 \mathrm{MHz}$ requires a bath temperature below about $5 \mathrm{mK}$ for a thermal occupation less than unity. Such temperatures are achievable but challenging with cryogenic cooling [56]. At a more accessible cryostat temperature of $33 \mathrm{mK}$, the initial thermal occupation is $\bar{n}=5$. To approach the ground state from a thermal state, we propose a cooling scheme utilizing the qubit as a thermal filter (Fig. 2). Following initialization to the $|+\rangle$ state, the
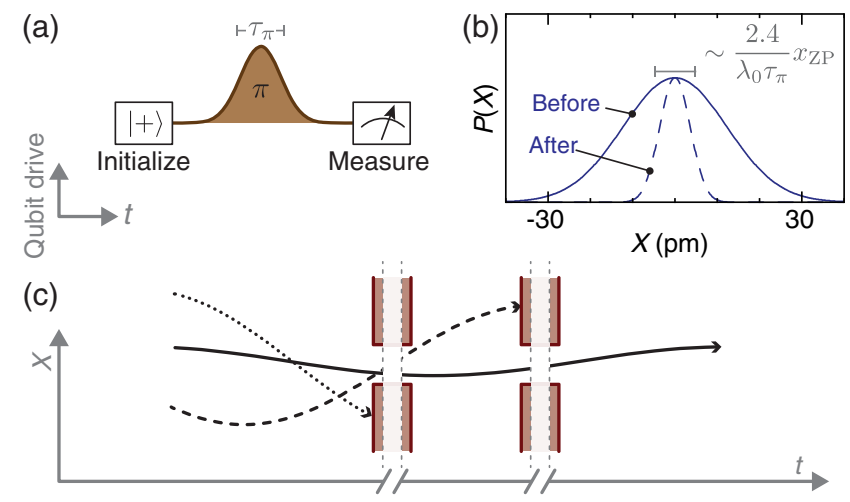

FIG. 2. Cooling the resonator using the qubit. (a) Pulse sequence (see text). Gaussian pulse shaping is chosen for near-optimal filtering. (b) Effect of this sequence, conditioned on the qubit outcome $|-\rangle$, on an initial mechanical distribution with a thermal distribution $\bar{n}=5$. (Burst duration is $\tau_{\pi}=38 \mathrm{~ns}$ with $\lambda_{0} / 2 \pi=4.2 \mathrm{MHz}$.) The pulse sequence has the effect of passing the wave function through a narrow slit. The filter function is obtained by numerically solving the time evolution generated by Eq. (5) with an additional $\pi$-pulse term $g(t) \sigma_{x} / 2$. (c) Cartoon showing the effect of two pulse sequences, offset by a quarter of a mechanical period. Only the lowest-energy trajectories (solid line) survive both measurements, effectively cooling the resonator. For the purpose of this cartoon, the duration of the two pulse sequences is compressed; in fact, each pulse sequence lasts for several mechanical periods. scheme consists of applying a $\pi$ burst at the bare qubit frequency $\omega_{q}$ [Fig. 2(a)]. If the resonator is near its equilibrium position, this results in a qubit flip. By conditioning on this outcome (i.e., utilizing only those runs of the experiment where this qubit outcome is measured), the resonator state is constrained to a narrow window [Fig. 2(b)].

A single operation of this type cools only one quadrature of the motion because resonator states with high momentum may still pass the window. To cool the orthogonal quadrature, the same selection should be applied a quarter of a mechanical period later [57], which filters out highenergy states that pass the first selection step [Fig. 2(c)]. The combination of these two pulse sequences therefore prepares the resonator close to its ground state, at the price of accepting only a fraction of the measurement runs.

\section{B. Diffracting the resonator}

The effective diffraction grating for the resonator (Fig. 3) is implemented using Ramsey interferometry to generate a periodic spatial filter $[58,59]$. To understand how the grating arises, consider the time evolution operator $U(t)$ generated from Eq. (5). As shown in Ref. [59], this timeordered unitary is

$U(t)=\mathcal{R}(\Omega t) e^{-\left[\left(i \omega_{q} t\right) / 2\right] \sigma_{z}}\left(\mathcal{D}(\alpha)|-\rangle\left\langle-\left|+\mathcal{D}^{\dagger}(\alpha)\right|+\right\rangle\langle+|\right)$,

where $\mathcal{D}(\alpha)=e^{\alpha a^{\dagger}-\alpha^{*} a}$ and $\mathcal{R}(\theta)=e^{-i \theta a^{\dagger} a}$ are resonator displacement and rotation operators, respectively, and

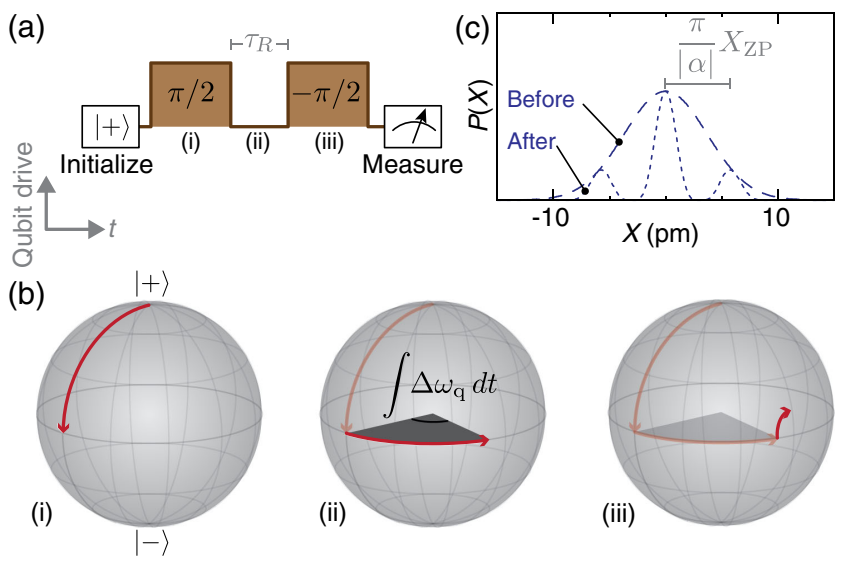

FIG. 3. Using pulsed qubit operations to construct an effective diffraction grating. (a) Pulse sequence. (b) Evolution of the qubit on the Bloch sphere, during (i) preparation, (ii) precession at a rate set by the displacement-dependent qubit frequency shift $\Delta \omega_{q}$, and (iii) projection. (c) Effect of this pulse sequence on the ground-state mechanical probability distribution. Conditioning on the qubit measurement outcome $|+\rangle$, the resonator wave function is multiplied by a periodic filter [Eq. (9)], analogous to a grating. Here, we have taken $|\alpha|=1.9$. 


$$
\alpha=i \int_{-\infty}^{t} e^{i \Omega t^{\prime}} \lambda\left(t^{\prime}\right) d t^{\prime}
$$

is the amplitude of the coherent displacement. The superposition of displacement operators in Eq. (6) applies equal and opposite momentum kicks [assuming $\operatorname{Re}(\alpha)=0$ from here on [60]] to the resonator depending on the state of the qubit. This is analogous to standing-wave gratings in atom interferometry, which, when decomposed into left- and right-propagating beams, can be understood to impart superposed positive and negative impulses to atoms.

We now describe the protocol that realizes this superposition of momentum kicks [Figs. 3(a) and 3(b)]. Following initialization of the qubit in the excited state $|+\rangle$, a microwave burst applied to the qubit generates a $\pi / 2$ rotation, preparing a superposition $(|+\rangle+|-\rangle) / \sqrt{2}$. The mechanical interaction then causes the qubit state to precess at the displacement-dependent rate $\Delta \omega_{q}(t)=$ $\left\{2 X(t) / X_{\mathrm{ZP}}\right\} \lambda(t)$. After an interval $\tau_{R}$, a second $\pi / 2$ burst is applied, followed by a $\sigma_{z}$ measurement. Conditioning on the qubit outcome gives a measurement operator that acts on the mechanical system,

$$
\begin{aligned}
\Upsilon_{ \pm}(\phi) & \equiv\left\langle \pm\left|\Pi_{\phi}^{\dagger} U(t) \Pi_{0}\right|+\right\rangle \\
& =\frac{\mathcal{R}(\Omega t)}{2}\left[\mathcal{D}^{\dagger}(\alpha) \pm e^{i \phi+i \omega_{q} t} \mathcal{D}(\alpha)\right] .
\end{aligned}
$$

Here, $\Pi_{\phi}$ denotes a $\pi / 2$ qubit rotation with phase $\phi$, and \pm is the result of the $\sigma_{z}$ measurement. The (unnormalized) state of the resonator after the interaction is

$|\psi\rangle_{M} \rightarrow \Upsilon_{ \pm}|\psi\rangle_{M}=\mathcal{R}(\Omega t) \underset{\sin }{\cos }\left(|\alpha| \frac{X}{X_{\mathrm{ZP}}}+\frac{\phi}{2}\right)|\psi\rangle_{M}$,

where $\cos (\cdot)$ or $\sin (\cdot)$ correspond to finding the qubit in the excited or ground state, respectively [61]. The resonator wave function is thus projected onto an effective diffraction grating with pitch $\pi X_{\mathrm{ZP}} /|\alpha|$ [Fig. 3(c)]. Since the only difference between conditioning on the $| \pm\rangle$ outcomes is a relative change in phase of the effective grating, either measurement outcome may be used to define the grating. We refer to the Ramsey sequence followed by conditioning on the qubit measurement outcome as a grating operation. Its effect is to split the resonator wave function into a superposition of left-moving and right-moving branches.

A well-separated superposition, with both branches displaced by more than the zero-point amplitude, requires $|\alpha| \gtrsim 1$. To achieve this with our parameters, we require that $\lambda(t)$ is modulated at the mechanical frequency:

$$
\lambda(t)=\lambda_{0} g(t) \cos \Omega t,
$$

where $g(t)$ is a Gaussian envelope function with a maximum of unity and a full width at half maximum of $\tau_{\lambda} \gg 1 / \Omega$. Equation (7) then gives

$$
\alpha=i \frac{\sqrt{\pi}}{4 \sqrt{\ln 2}} \lambda_{0} \tau_{\lambda}
$$

In the following, we take $\alpha \approx 1.9 i$, thus achieving the desired momentum separation. A price to pay for this modulation is that the qubit and resonator are susceptible to decoherence over the full duration of the envelope. With our parameters, we require $\tau_{\lambda} \approx 130 \mathrm{~ns}$, corresponding to $N \approx 17$ mechanical periods. This interaction time is short enough that the evolution of the resonator-qubit system is well approximated as unitary. (See Appendix C for modeling of qubit dephasing and mechanical decoherence.)

\section{Nanomechanical interferometry}

We now show how a sequence of grating operations can be combined to create an interferometer (Fig. 4). The effect of

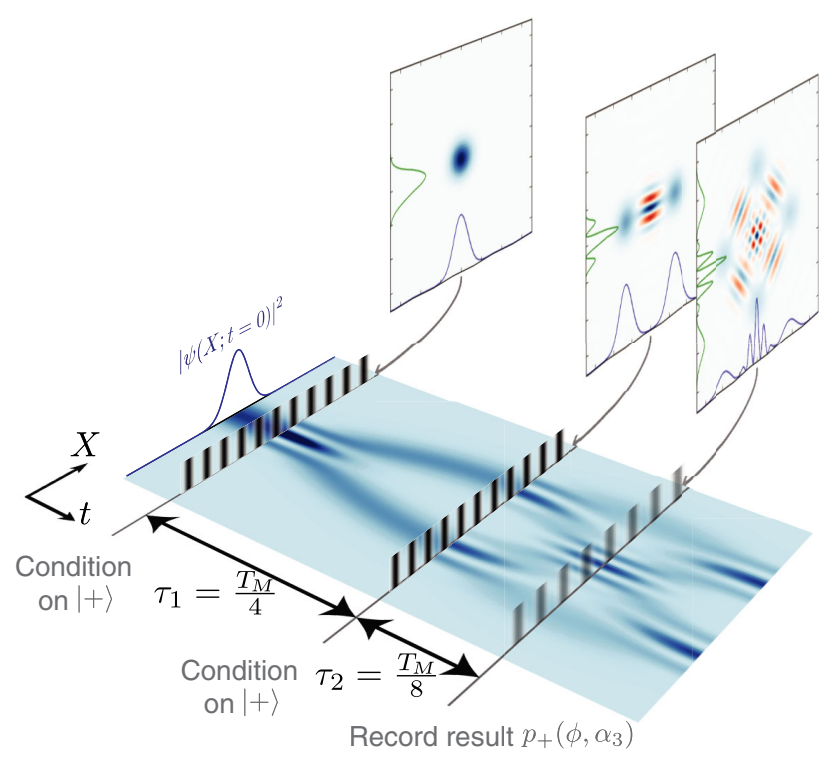

FIG. 4. Protocol for detecting nanomechanical interference. Following cooling (not shown), the first grating operation, with amplitude $\alpha_{1}$, diffracts the resonator wave function into a superposition of left-moving and right-moving components. After an interval $\tau_{1}$ of free evolution, here set as a quarter of the mechanical period $T_{M}$, a second grating operation is applied with amplitude $\alpha_{2}$. This leads to recombination of the two components an eighth of a mechanical period later. To measure the resulting interference, a third (unconditioned) Ramsey sequence is applied after time $\tau_{2}$ (here set to $T_{M} / 8$ ). The resulting qubit return probability $p_{+}\left(\alpha_{3}, \phi\right)$ probes the mechanical interference fringes. The main panel shows a simulated resonator spatial density $|\psi(X)|^{2}$, beginning from the ground state, plotted as a function of displacement and time, with the grating operations and the final Ramsey measurement operation indicated schematically as filters. The resonator Wigner distributions and marginals (see Fig. 5) are shown as insets just before each filter. To illustrate the continuing periodic evolution of the resonator wave function, the spatial density beyond the final Ramsey measurement is plotted as it would be probed by applying the measurement instead at a later time. 
a single grating operation [Eq. (9)] with phase $\phi=0$ and amplitude $\alpha=\alpha_{1}$ is to divide the resonator's wave function into two components with added momentum $\pm\left|\alpha_{1}\right| \hbar / X_{\mathrm{ZP}}$. A second grating operation with the same amplitude and phase, applied after a duration $\tau_{1}=\pi / 2 \Omega$ corresponding to a quarter period of free evolution, further splits the branches of the superposition, allowing quantum interference between recombined branches to be observed. After a second evolution time $\tau_{2}$, the interference can be detected using a third Ramsey sequence. In this step, there is no conditioning; the probability $p_{+}\left(\phi, \alpha_{3}\right)$ for the qubit to return to state $|+\rangle$ is measured as a function of the phase $\phi$ and amplitude $\alpha_{3}$ of the Ramsey sequence. This probability is

$$
\begin{aligned}
p_{+}\left(\phi, \alpha_{3}\right) & =\operatorname{Tr}\left[\Upsilon_{+}^{\dagger} \Upsilon_{+} \rho_{m}\right] \\
& =\int d X^{\prime} \cos ^{2}\left(\left|\alpha_{3}\right| \frac{X^{\prime}}{X_{\mathrm{ZP}}}+\frac{\phi}{2}\right) P\left(X^{\prime}\right),
\end{aligned}
$$

where $\rho_{m}$ is the density matrix describing the state of the resonator immediately before the third grating, with position probability distribution $P(X)$.

Scanning the phase of the third Ramsey sequence is analogous to scanning the position of the third grating in a molecular interferometer [2], and the signature of interference is a sinusoidal dependence on $\phi$. In fact, Eq. (12) can be understood as a Fourier decomposition in which each choice of $\left|\alpha_{3}\right|$ probes the component of $P(X)$ with wave number $2\left|\alpha_{3}\right|$. From here on, we use $x \equiv X / X_{\mathrm{ZP}}=(a+$ $\left.a^{\dagger}\right)$ as a dimensionless position operator.

Our goal now is to use $p_{+}$to distinguish quantum interference from classical fringes that might appear in the resonator's probability distribution $P(x)$. Classical fringes might arise, for example, from the shadow of the diffraction gratings, or from Moiré patterns. To recognize the quantum interference, we plot the resonator Wigner distribution at different times $\tau_{2}$ after the second grating operation, choosing $\left|\alpha_{1}\right|=\left|\alpha_{2}\right| \approx 1.9$ (Fig. 5). The effect of applying the first grating operation is to "slice" the position distribution by multiplying by $\cos ^{2}(|\alpha| x)$ and to prepare a superposition of two momentum states (see second inset in Fig. 4, plotting the state after the first grating, rotated by one quarter period). Since the second grating operation is applied a quarter period after the first, it acts along an orthogonal axis, leading to the "quantum compass" state of Fig. 5(a) [62]. These compass states are widely studied theoretically and find applications in, e.g., sensing and metrology [63] and quantum codes with multicomponent superpositions [64].

The compass state can be intuitively understood as follows. The quarter-period rotation after the first grating turns the momentum superposition state into a position superposition. Each branch of the superposition then passes the grating, generating its own momentum superpositions and resulting in the four-lobe compass state. The compass state is clearly visible if the resonator is initially prepared in

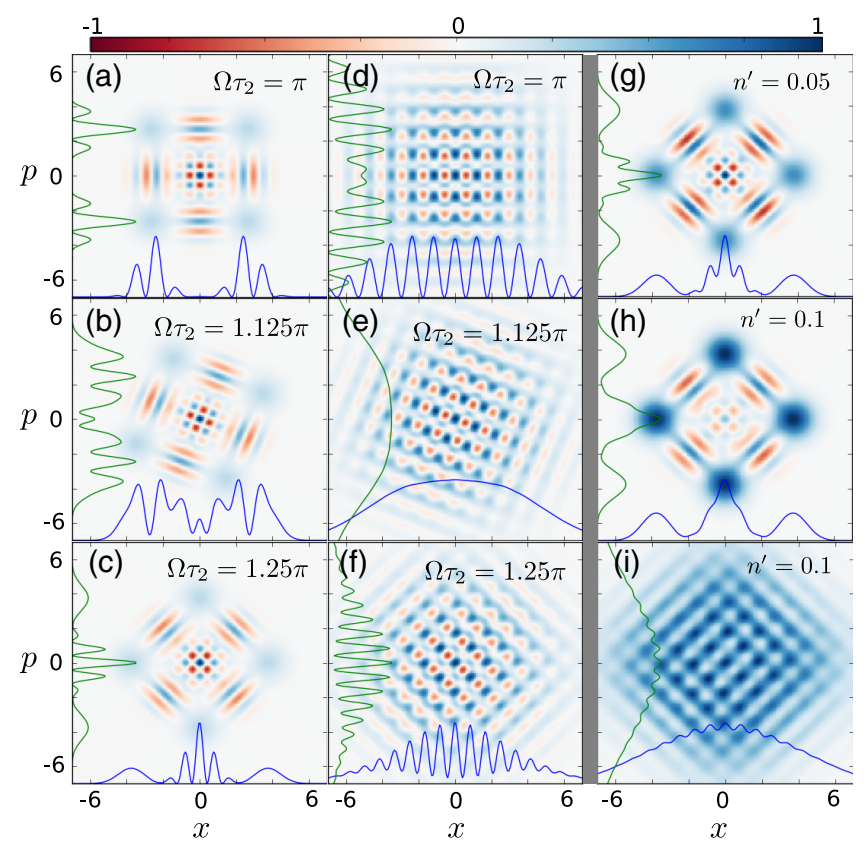

FIG. 5. Wigner distributions of the resonator's state at different values of $\tau_{2}$. (a)-(c) Maximum interference from an initial pure state with unitary evolution. (d)-(f) Loss of interference due to initial thermal phonon occupation $\bar{n}=5$ (with unitary evolution). (g)-(i) Loss of interference with nonunitary evolution equivalent to adding $0.05(\mathrm{~g})$ or $0.1(\mathrm{~h}, \mathrm{i})$ thermal phonons. The initial state for panels $(\mathrm{g})$ and $(\mathrm{h})$ is the vacuum state, and the initial state of panel (i) is a thermal state with $\bar{n}=5$ phonons. Blue and green traces show the position and momentum marginals, respectively, normalized to the same maximum. Each plot uses $|\alpha| \approx 1.9$, and the color scale has been normalized to the range \pm 1 .

the ground state, $\bar{n}=0$ [Figs. 5(a)-5(c)], but is washed out if the resonator is initially in a thermal state, leaving only the orthogonally sliced pattern visible [Figs. 5(d)-5(f)]. During the evolution time $\tau_{2}$, the Wigner distribution rotates [Figs. 5(b), 5(c), 5(e), and 5(f)] so that the interference fringes oscillate between the position and momentum marginals (plotted as blue and green traces, respectively). Interference patterns arise when two lobes of a coherent quantum superposition overlap in position space, for example, the northeast and southeast lobes in Fig. 5(a) interfering around $x \approx 3$, or the north and south lobes in Fig. 5(c) interfering around $x \approx 0$. The wave numbers present in the position marginal (which is measured by the third grating) are proportional to the momentum separation of lobes in phase space, geometrically illustrating the $\sqrt{2}$ ratio between wave-number components present in Figs. 5(a) and 5(c).

The interference fringes arising when the resonator is initially prepared in its ground state can be compared with those arising from an initial thermal state $(\bar{n}=5)$. If the width of the initial thermal state [as in Figs. 5(d)-5(f)] is larger than the superposition size $(\sqrt{\bar{n}}>|\alpha|)$, then the vertical slicing of the grating is no longer accompanied by a 
distinct momentum superposition but rather by an increase in the momentum variance [as seen by the broaderthan-Gaussian position and momentum distributions in Fig. 5(e)], and this results in a checkerboard pattern.

We can now see the distinction between quantum and classical fringes appearing in the marginal distributions. The shadow of the gratings is dominated by components close to the wave number $2\left|\alpha_{1}\right|$ [Figs. 5(a) and 5(d)]. The Moiré patterns arising from the checkerboard have components close to at most two wave numbers, $2\left|\alpha_{1}\right|$ and $2 \sqrt{2}\left|\alpha_{1}\right|$ (see Appendix D). By contrast, the quantum interference pattern [Figs. 5(a)-5(c)] has multiple wavenumbers components at each $\Omega \tau_{2}$, as seen in the position marginals. Furthermore, quantum interference appears for all evolution times $\tau_{2}$, whereas classical fringes are washed out [Fig. 5(e)] except at particular fractions of the mechanical period. Hence, for this protocol, a marginal $P(x)$ with multiple wave-number components, observed at all rotation angles $\Omega \tau_{2}$, indicates quantum interference.

We now show that this interferometer is a sensitive probe for quantum decoherence, which damps the interference fringes in $P(x)$ and therefore destroys the signature of quantum coherence in $p_{+}$. To model decoherence following the second grating operation, we consider weak thermalization of the state, resulting in a decohered state (superscript d),

$$
\rho_{m}^{(d)}=\int d^{2} \beta \frac{e^{-|\beta|^{2} / n^{\prime}}}{\pi n^{\prime}} \mathcal{D}(\beta) \rho_{m} \mathcal{D}^{\dagger}(\beta),
$$

where $n^{\prime}$ is the number of thermal phonons effectively added to the resonator, causing loss of quantum coherence. The loss of coherence is equivalent to convolving $P(x)$ with a Gaussian of width $\sqrt{n^{\prime}}$, thereby exponentially damping oscillations of wave number $|\alpha|>\sqrt{n^{\prime}}$ (Appendix C). Figures 5(g)-5(i) plot the effect of loss of coherence between the second and third gratings, assuming an initial ground state $(\mathrm{g})$ and $(\mathrm{h})$, and an initial $(\bar{n}=5)$ thermal state (i). The decoherence is modeled only after the second grating, so without thermalization, panels (g) and (h) would coincide with panel (c), and panel (i) would coincide with panel (f). The plots show that even the addition of a fraction of a phonon drastically suppresses the interference pattern in $P(x)$ and the corresponding signature in $p_{+}$.

Finally, in Fig. 6, we show explicitly how these two effects - thermal occupation before the interferometry and decoherence during the interferometry-degrade the quantum signatures in $p_{+}$. In the ideal case [Fig. 6(a)], there are several values of $\alpha_{3}$ at each time $\tau_{2}$ that give nontrivial probabilities of $p_{+}$. In contrast, beginning in a thermal state with $\bar{n}=5$ phonons [Fig. 6 (b)], all fringes are washed out, with the exception of the shadow of the grating (at $\alpha_{3} / \alpha_{2}=$ 1 and $\Omega \tau_{2}=0, \pi / 2$ ) and the Moiré pattern (at $\alpha_{3} / \alpha_{2}=\sqrt{2}$ and $\left.\Omega \tau_{2}=\pi / 4\right)$. Loss of coherence during the interferometry [Fig. 6(c)] leads to a qualitatively different behavior,

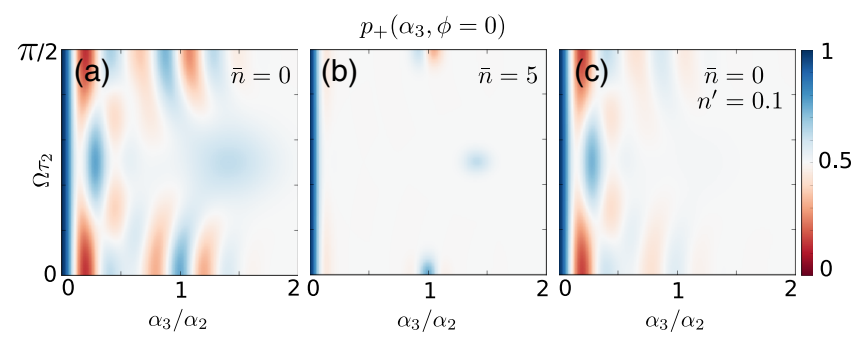

FIG. 6. The probability to find the qubit in its excited state, $p_{+}\left(\alpha_{3}\right)$, can be used to probe different wave-number components in the position probability distribution $P(x)$. As the resonator evolves (increasing $\Omega \tau_{2}$ ), different wave numbers are present in $P(x)$ (see Fig. 5). The probability $p_{+}\left(\alpha_{3}\right)$ is plotted for the resonator initially in (a) a pure state, (b) a thermal state of $\bar{n}=5$ phonons, and (c) a pure state, but with decoherence (represented by $n^{\prime}$ added phonons) after the second grating.

namely, damping of all features in $p_{+}$, including the classical fringes, for $\left|\alpha_{3}\right|>\sqrt{n^{\prime}}$. This mechanical interferometry scheme could thus be used as a specific probe of quantum decoherence during the mechanical evolution.

We can compare the degree of macroscopicity in this proposal with other quantum superposition experiments. With parameters from Appendix A, a $3 \times 10^{6}$ amu resonator achieves a maximum spatial superposition of $12 \mathrm{pm}$. Applying the macroscopicity measure $\mu$ introduced in Ref. [65], the nanotube reaches $\mu=14.0$ (assuming a fringe visibility of 0.8 and coherence time of 100 oscillator periods). By this measure, the superposition is more macroscopic than already achieved in state-of-the-art near-field molecular interferometry experiments [66] $(\mu=12.1)$ or in a micromembrane near the ground state [17] $(\mu=11.5)$, and it is comparable to other proposals, including a satellite-based atom interferometer [67] $(\mu=14.5)$ or a $10^{5}$ amu Talbot-Lau interferometer [68] $(\mu=14.5)$. However, because of its low mass, a nanotube has a lower measure than proposals for superpositions of a micromirror [9] ( $\mu=19.0)$ or nanosphere interference [10] $(\mu=20.5)$ [69].

Before concluding, we contrast our scheme with conventional optical interferometers and current matter-wave interferometers. The interference fringes of Fig. 5(c) are loosely analogous to Mach-Zehnder interference because the mechanical wave function is split and then recombined. However, the full position distribution is a result of all four components of the superposition state, whereas conventional interferometers usually combine only two optical paths. In matter-wave experiments, a successful current technique is Talbot-Lau interferometry [2], where a sequence of gratings is used to generate and observe near-field diffraction patterns. In our scheme, the quarter period of mechanical free evolution between the gratings is equivalent to far-field diffraction, so the key physics is quite different. We further highlight that the mechanical state prepared after the second grating is the "quantum compass 
state" introduced by Zurek [62]; this scheme allows the features of this exotic quantum state to be explored. Furthermore, this work opens an avenue to implement other pulse sequences that create grating geometries for mechanical-state engineering.

\section{CONCLUSION}

We have introduced the displacemon electromechanical system, which provides sufficiently strong coupling to generate and detect quantum interference of a massive object containing a quarter of a million atoms. Using device parameters based on current technology, our qubit-resonator displacemon can achieve effective ultrastrong coupling using a modulated coupling scheme. A similar interferometry scheme could also be applied to other kinds of solid-state qubits coupled to high-quality resonators, such as spin qubits coupled to nanotubes [32,33], diamond defects coupled to cantilevers [55], or piezoelectric resonators coupled to superconducting qubits [70,71]. However, the parameters estimated for the proposed device of Fig. 1 may be particularly experimentally favorable for this implementation because they imply that the coupling exceeds both the thermal decay rate of the resonator and the typical dephasing rate of a qubit (see Appendix E). Importantly, our scheme does not rely on degeneracy between the qubit and the resonator [15], nor on qubit coherence over the lifetime of the mechanical superposition [72].

Using this ultrastrong coupling, we have shown that it is possible to extend matter-wave interferometry to nanomechanical resonators, opening up a range of new devices that can be used to study quantum physics at the mesoscale. Furthermore, interferometry performed on the wave function of a mechanically bound resonator is qualitatively distinct from existing free-particle interferometry techniques. An important advantage of nanomechanical resonators for quantum tests is that they can readily be extended to probe much larger masses than in molecular vapors or even levitated nanoparticles. Although the nanotube resonator considered here does not have enough mass to seriously challenge the interesting parameter regime of objective collapse theories, a similar protocol could be extended to more massive objects still well within the range of nanomechanics. This research direction could allow for testing specific theories of quantum collapse [8], as an alternative to proposals based on single-photon optomechanics [9] or levitated nanoparticles [11,72]. Multiple resonators coupled to the same qubit (such as the pair of nanotube junctions in Fig. 1) could allow for generation of entanglement between massive objects, leading to Bell tests of mechanical resonators [73].

\section{ACKNOWLEDGMENTS}

We thank P. J. Leek and E. M. Gauger for discussions. We acknowledge FQXi, EPSRC (EP/N014995/1), Templeton
World Charity Foundation, and the Royal Academy of Engineering. K. K. would like to thank the Department of Materials, University of Oxford for their hospitality during the initial stages of this work.

\section{APPENDIX A: DEVICE PARAMETERS AND IMPLEMENTATION}

We assume device parameters based on a mixture of experiment and simulation as follows. We take the parameters of the nanotube and the junctions from the nanotube SQUID device of Ref. [43]. For a resonator with length $l=800 \mathrm{~nm}$, the frequency was measured as $\Omega / 2 \pi=$ $125 \mathrm{MHz}$, which, with estimated diameter $D=2.5 \mathrm{~nm}$ and mass $m=5 \times 10^{-21} \mathrm{~kg}=3 \times 10^{6} \mathrm{amu}$, leads to $X_{\mathrm{ZP}}=$ $3.7 \mathrm{pm}$, typical of nanotube resonators. In each SQUID junction, a critical current $I_{c} \approx 12 \mathrm{nA}$ was achieved, implying $E_{J}^{0} / h=12 \mathrm{GHz}$.

For the qubit, the charging energy is set by the electrode geometry, which is a design choice. Finite-element capacitance simulation for the device of Fig. 1(b), with qubit diameter $340 \mu \mathrm{m}$, gives $E_{C} / h=0.2 \mathrm{GHz}$, typical of qubit devices and well into the transmon regime $E_{J}^{0} \gg E_{C}$ [74]. The maximal qubit frequency is then $\omega_{q}^{0} / 2 \pi=$ $\sqrt{8 E_{J} E_{C}} / h=4.4 \mathrm{GHz}$, and the calculated qubit energy levels are shown in Fig. 1(c).

For the in-plane magnetic field, we assume $B_{\|}=0.5 \mathrm{~T}$, which nanotube SQUIDs can withstand [43]. The operating flux point should then be chosen to maximize $\lambda$ while still maintaining a qubit frequency compatible with microwave resonators. We assume flux bias $\Delta \Phi / \Phi_{0}=-0.84$, leading to a qubit frequency $\omega_{q}=2 \pi \times 2.19 \mathrm{GHz}=\omega_{q}^{0} / 2$ [dashed vertical line in Figs. 1(c) and 1(d)]. With symmetric junctions, and assuming that the restoring force on the nanotube is dominated by tension, the coupling is then $\lambda / 2 \pi=4.2 \mathrm{MHz}$. Since the coupling can be reduced by tuning $\Delta \Phi$ towards zero, we take this as the maximum coupling strength $\lambda_{0}$.

In a realistic device, we must take account of asymmetry between the junctions. Denoting the two critical currents by $I_{c 1}$ and $I_{c 2}$, with $\delta \equiv 2\left(I_{c 1}-I_{c 2}\right) /\left(I_{c 1}+I_{c 2}\right)$ being the asymmetry parameter, we have $E_{J}=$ $E_{J}^{0} \sqrt{\cos ^{2}\left(\pi \Delta \Phi / 2 \Phi_{0}\right)+\left(\delta^{2} / 4\right) \sin ^{2}\left(\pi \Delta \Phi / 2 \Phi_{0}\right)}$, with a corresponding modification to Eq. (4) [47,52]. This asymmetry leads to a small reduction in $\lambda_{0}$ [Figs. 1(c) and 1(d)].

For these parameters, the device would be in the strong coupling regime $\left(\lambda_{0}>k_{B} T / \hbar Q_{m}, 1 / T_{2}\right)$ for a comparatively modest resonator quality factor $Q_{m} \gtrsim 15$ and $T_{2} \gtrsim 120 \mathrm{~ns}$. Accessing the ultrastrong coupling regime $\left(\lambda_{0}>\Omega\right)$ is more challenging but may be possible [47]: If the suspended length could be increased to $l \approx 2.9 \mu \mathrm{m}$ and the tension reduced to zero while keeping other parameters unchanged, the coupling would be $\lambda_{0} / 2 \pi \approx \Omega / 2 \pi \approx$ $8 \mathrm{MHz}$. However, in the simulations, we do not make this 
assumption but instead assume that effective ultrastrong coupling is engineered by toggling $\lambda(t)$ as in Eq. (10).

Previous experiments have measured carbon nanotube resonators at temperatures smaller than the level spacing and therefore presumably in the quantum ground state $[39,40]$. However, those experiments did not measure quantum effects. In this proposal, the strongly coupled qubit provides the nonlinearity that allows non-Gaussian states to be prepared and detected, in a way that goes beyond measurements using resistive transport [38], SQUID measurements [43], or linear optomechanics [50]. The proposed qubit imposes important experimental challenges. The tunnel coupling between nanotube and superconductor must be large to support a high critical current. Furthermore, both the superconducting film and the nanotube junction must remain superconducting even in an in-plane magnetic field, and the qubit must remain coherent. The modulated electromechanical coupling scheme considered here requires precise timing of the flux bias pulses to avoid phase errors. However, experiments are making progress in these directions: dc transport measurements show that a suspended nanotube SQUID can be operated up to magnetic field $B_{\|}=1 \mathrm{~T}$ [43]. Meanwhile, pulsed flux bias is an established tool for controlling transmon qubits $[37,54]$.

\section{APPENDIX B: COOLING VIA QUBIT MEASUREMENT}

Here, we show that the $\sigma_{z}\left(a+a^{\dagger}\right)$ interaction Hamiltonian can be used to conditionally cool the resonator. Consider the qubit-resonator interaction in the interaction picture, with a (real) coherent qubit drive $g(t)$ with zero detuning to the qubit frequency,

$$
H_{I} / \hbar=\lambda(t) \sigma_{z}\left(a+a^{\dagger}\right)+g(t) \sigma_{x} .
$$

The cooling protocol works as follows: First, the qubit is prepared in the $|-\rangle$ eigenstate; then, the interaction is switched on $\left(\lambda(t) \rightarrow \lambda_{0}\right)$ and remains time independent for the duration of the $\pi$ pulse $g(t)$. At the completion of the $\pi$ pulse, the interaction is switched off, and the qubit is projectively measured in the $\sigma_{z}$ basis.

The $\pi$-pulse cooling can be intuitively understood. If the resonator remains at a fixed position over the duration of the $\pi$ pulse (as it must in the interaction picture since $a+a^{\dagger}$ commutes with the Hamiltonian), then the $\sigma_{z}$ term in Eq. (B1) looks like a detuning of the $\pi$-pulse drive from the qubit frequency. The probability of a qubit transition from the ground to the excited state under a $\pi$ pulse depends on the detuning and is maximized for zero detuning. Therefore, if the resonator is close to its equilibrium position, then there is a high probability of the qubit transitioning to the $|+\rangle$ state. However, if the resonator is far displaced from equilibrium (i.e., in a high potential energy state), then the qubit has a low transition probability.
In the following, we describe how the cooling envelope in Fig. 2 is obtained. Consider the state of a pure qubitresonator system,

$$
|\psi(t)\rangle=\int c_{-}\left(x^{\prime}, t\right)\left|-, x^{\prime}\right\rangle+c_{+}\left(x^{\prime}, t\right)\left|+, x^{\prime}\right\rangle d x^{\prime},
$$

where $c_{-}\left(x^{\prime}, t\right)$ is the probability amplitude of finding the qubit in the $|-\rangle$ state and the resonator at $x^{\prime}$. The Hamiltonian Eq. (B1) generates the equations of motion,

$$
\begin{aligned}
& \dot{c}_{+}(x, t)=-i \lambda_{0} x c_{+}(x, t)-i g(t) c_{-}(x, t), \\
& \dot{c}_{-}(x, t)=-i \lambda_{0} x c_{-}(x, t)-i g(t) c_{+}(x, t) .
\end{aligned}
$$

The conditional state of the resonator after the cooling measurement (with outcome $|+\rangle$ ) is therefore $|\psi\rangle_{m} \propto$ $\int d x^{\prime} c_{+}\left(x^{\prime}, t_{f}\right)|x\rangle$, where $c_{+}\left(x^{\prime}, t_{f}\right)$ is the solution to Eqs. (B3) and (B4) after the application of the $\pi$ pulse, with initial conditions $c_{-}(x, 0)=\langle x \mid \psi(0)\rangle_{m}$ and $c_{+}(x, 0)=0$ (i.e., the qubit is initially in the $|-\rangle$ state). The $\alpha$ is used since the final state is not normalized. This generalizes to an initial mixed state of the resonator by

$$
\begin{gathered}
\rho(0)=|-\rangle\left\langle-\left|\otimes \sum_{i} p_{i}\right| \psi_{i}\right\rangle_{m}\left\langle\left.\psi_{i}\right|_{m} \rightarrow\right. \\
\rho_{m}\left(t_{f}\right) \propto \sum_{i} p_{i} \int d x^{\prime} d x^{\prime \prime} c_{+, i}\left(x^{\prime}\right)\left|x^{\prime}\right\rangle\left\langle x^{\prime \prime}\right| c_{+, i}^{*}\left(x^{\prime \prime}\right),
\end{gathered}
$$

where $p_{i}$ is the classical probability of finding the quantum system in the state $\left|\psi_{i}\right\rangle_{m}$. The calculation can be greatly simplified by noting that the system of differential equations governing $c_{ \pm}$is linear, and therefore, the solution for the initial conditions $c_{-}=1, c_{+}=0$ can be used to find $c_{ \pm, i}\left(x^{\prime}, t_{f}\right)$ for any initial state.

The cooling envelope in Fig. 2 is obtained by numerically solving Eqs. (B3) and (B4), with $g(t)$ a Gaussian envelope $\pi$ pulse, and then calculating the new position marginal,

$$
\left\langle x\left|\rho_{m}\left(t_{f}\right)\right| x\right\rangle \propto \sum_{i} p_{i}\left|c_{+, i}(x)\right|^{2}
$$

where $\rho_{m}(0)$ is a thermal state.

\section{APPENDIX C: DECOHERENCE}

Here, we model the effect of decoherence on the interference. For our chosen parameters, the effect is estimated to be weak because the interaction time $\tau_{\lambda} \approx 130 \mathrm{~ns}$ is short compared with other timescales. For a superconducting qubit, the decoherence time is typically $T_{2}>1 \mu \mathrm{s} \gg \tau_{\lambda}$, so qubit dephasing during the interaction will not significantly change the state. For the resonator, the high quality factor $Q_{m}$ suppresses thermal decoherence; assuming $Q_{m}=10^{5}$, there 
are $\bar{n} / Q_{m} \approx 5 \times 10^{-5}$ phonons exchanged with the thermal environment every resonator period, or 1 phonon exchanged about every $10^{3}$ realizations of the interaction. Below, we model the effect of decoherence in detail.

\section{Qubit dephasing}

We model dephasing by adding a stochastic frequency shift to the qubit, changing the Hamiltonian [Eq. (5)] to

$$
H=\hbar \Omega a^{\dagger} a+\hbar \frac{\omega_{q}}{2} \sigma_{z}+\hbar \lambda(t)\left(a+a^{\dagger}\right) \sigma_{z}+\hbar \sqrt{\gamma / 2} \xi(t) \sigma_{z},
$$

where $\gamma$ is the qubit dephasing rate and $\xi(t)$ is a deltacorrelated white noise term satisfying $\mathcal{E}(\xi(t))=0$ and $\mathcal{E}\left(\xi(t) \xi\left(t^{\prime}\right)\right)=\delta\left(t-t^{\prime}\right)$. Here, $\mathcal{E}(\cdot)$ denotes an average over realizations of this stochastic process. Moving into the interaction picture, the unitary generated by this Hamiltonian is

$U(t)=e^{-i W(t) \sqrt{\gamma / 2} \sigma_{z}}\left(\mathcal{D}(\alpha)|-\rangle\left\langle-\left|+\mathcal{D}^{\dagger}(\alpha)\right|+\right\rangle\langle+|\right)$,

where $W(t)=\int_{t_{0}}^{t} \xi\left(t^{\prime}\right) d t^{\prime}$ is a stochastic variable with a mean of zero and a variance of $t$. Since there is classical uncertainty in the realization of $W(t)$, the joint state of the resonator-qubit system will be mixed. Because of this classical uncertainty, the measurement operator cannot be understood as mapping pure states to pure states as assumed in Eq. (8).

We must therefore consider the measurement procedure (used to impose the grating) in the density matrix description. Before switching the interaction on, i.e., while $\lambda(t)=0$, the $\pi / 2$ pulse changes the $|+\rangle\langle+|$ state of the qubit to $\rho_{q}=\frac{1}{4}(|+\rangle+|-\rangle)(\langle+|+\langle-|)$. The state of the mechanical resonator is left unchanged in an arbitrary state $\rho_{m}$. This joint state must be separable because the initialization of the qubit state at the beginning of the grating operation has the effect of destroying any qubit-resonator entanglement.

As the interaction is switched on, the joint state of the system evolves according to

$$
\begin{aligned}
\rho(t, W(t))= & U(t) \rho_{q} \otimes \rho_{m} U^{\dagger}(t) \\
= & \frac{1}{2} e^{2 i \sqrt{\gamma / 2} W(t)}|-\rangle\langle+| \otimes \mathcal{D}(\alpha) \rho_{m} \mathcal{D}(\alpha) \\
& +\frac{1}{2} e^{-2 i \sqrt{\gamma / 2} W(t)}|+\rangle\langle-| \otimes \mathcal{D}^{\dagger}(\alpha) \rho_{m} \mathcal{D}^{\dagger}(\alpha) \\
& +\frac{1}{2}|+\rangle\langle+| \otimes \mathcal{D}^{\dagger}(\alpha) \rho_{m} \mathcal{D}(\alpha) \\
& +\frac{1}{2}|-\rangle\langle-| \otimes \mathcal{D}(\alpha) \rho_{m} \mathcal{D}^{\dagger}(\alpha),
\end{aligned}
$$

where $U(t)$ is the unitary operator in Eq. (C2). Since $W(t)$ is unknown, the resulting quantum state at time $t$ must be weighted by the probability of obtaining a particular realization of $W(t)$, where $P(W(t))=\exp \left[-W^{2}(t) / 2 t\right] / \sqrt{2 \pi t}$,

$$
\rho(t)=\int_{-\infty}^{\infty} \rho(t, W(t)) P(W(t)) d W(t) .
$$

Projecting the qubit onto the state $\left(|+\rangle+e^{-i \phi}|-\rangle\right) / \sqrt{2}$ gives the unnormalized conditional state of the mechanical resonator,

$$
\begin{aligned}
& \rho_{m, \pm} \sim \frac{1}{2}\left(\left\langle+\left| \pm e^{i \phi}\langle-|\right) \rho(t)\left(|+\rangle \pm e^{-i \phi}|-\rangle\right)\right.\right. \\
& \sim \frac{1}{4}\left[\mathcal{D}^{\dagger}(\alpha) \rho_{m} \mathcal{D}(\alpha)+\mathcal{D}(\alpha) \rho_{m} \mathcal{D}^{\dagger}(\alpha)\right. \\
& \left.\quad \pm e^{-\gamma t}\left(e^{-i \phi} \mathcal{D}^{\dagger}(\alpha) \rho_{m} \mathcal{D}^{\dagger}(\alpha)+e^{i \phi} \mathcal{D}(\alpha) \rho_{m} \mathcal{D}(\alpha)\right)\right],
\end{aligned}
$$

where we have used " $\sim$ " because the right-hand side is unnormalized. Separating this into coherent and incoherent terms, we find

$$
\begin{aligned}
& \rho_{m, \pm} \sim \frac{e^{-\gamma t}}{4}\left[\mathcal{D}^{\dagger}(\alpha) \pm e^{i \phi} \mathcal{D}(\alpha)\right] \rho_{m}\left[\mathcal{D}(\alpha) \pm e^{-i \phi} \mathcal{D}^{\dagger}(\alpha)\right] \\
&+\frac{1-e^{-\gamma t}}{4}\left[\mathcal{D}^{\dagger}(\alpha) \rho_{m} \mathcal{D}(\alpha)+\mathcal{D}(\alpha) \rho_{m} \mathcal{D}^{\dagger}(\alpha)\right]
\end{aligned}
$$

We notice that the first term is proportional to $\Upsilon_{ \pm} \rho_{m} \Upsilon_{ \pm}^{\dagger}$, where $\Upsilon_{ \pm}$is given in Eq. (8) (with $\omega_{q}=0$ ). The first term in Eq. (C7) is exactly the state that one would expect if the grating protocol worked perfectly, while the second term is an incoherent mixture of displacements. We can therefore understand qubit dephasing as some classical probability that the resonator will coherently pass the grating and some probability that will we end up with an incoherent mixture. Since the normalization is state dependent, we cannot simply relate the coefficients in Eq. (C7) with direct probabilities. However, we can say that the relative probability of introducing an incoherent mixture is $\left(1-e^{-\gamma t}\right) / e^{-\gamma t}=e^{\gamma t}-1 \approx \gamma t$ for short times or a low dephasing rate. The trace of $\rho_{m, \pm}$ is the probability of finding the qubit in the $| \pm\rangle$ state, and using Eq. (C6), we may read off

$$
p_{ \pm}(\alpha, \phi)=\frac{1}{2} \pm \frac{e^{-\gamma t}}{4}\left[e^{i \phi} \chi(-2 \alpha)+e^{-i \phi} \chi(2 \alpha)\right],
$$

where $\chi(\cdot)=\operatorname{Tr}[\mathcal{D}(\cdot) \rho]$ is the characteristic function of the mechanical state [using $\operatorname{Tr}[\mathcal{D}(\alpha) \rho \mathcal{D}(\alpha)]=$ $\operatorname{Tr}[\mathcal{D}(\alpha) \mathcal{D}(\alpha) \rho]=\operatorname{Tr}[\mathcal{D}(2 \alpha) \rho]$, etc.]. The characteristic function is related to the Wigner distribution via a symplectic Fourier transform,

$$
\chi(\alpha)=\int d x d p W(x, p) e^{i x \alpha_{i}-i p \alpha_{r}},
$$

where $\alpha_{r(i)}$ is the real (imaginary) part of $\alpha$. If $\operatorname{Re}(\alpha)=0$, then 


$$
\begin{aligned}
\chi\left(2 \alpha_{i}\right) & =\int d x d p W(x, p) e^{2 i x \alpha_{i}} \\
& =\int d x P(x)\left[\cos \left(2 \alpha_{i} x\right)+i \sin \left(2 \alpha_{i} x\right)\right],
\end{aligned}
$$

where the complex part must vanish as $\chi(\cdot)$ is a real function (for states with $\pi$ rotational symmetry). This is simply the overlap integral between the position probability distribution $P(x)$ and a diffraction grating with a pitch $|\alpha|$. Therefore,

$p_{ \pm}(\alpha, \phi)=\frac{1}{2} \pm \frac{e^{-\gamma t} \int d x P(x) \cos (2 x|\alpha|)}{2} \cos (\phi)$,

which exactly probes the $2|\alpha|$ wave-number components in $P(x)$, with a reduced amplitude from the qubit dephasing.

Thus, we have seen that the effect of qubit dephasing is to introduce some probability of having an incoherent mixture of different momentum kicks, thus suppressing any signatures of interference in the outcomes of qubit measurements. Since the duration of the protocol is on the order of about $N$ mechanical oscillations, $t \approx 2 \pi \times N / \Omega$, to neglect qubit dephasing, we need $\gamma / \Omega \ll 1 / N$. For the parameters discussed in the main text, this requires $\gamma / 2 \pi<1 \mathrm{MHz}$.

\section{Loss of resonator coherence}

To see that oscillations in $p_{+}(|\alpha|, \phi)$ are a quantum effect, we consider the effect of adding $n^{\prime}$ thermal phonons to the state of the resonator immediately before the third grating [Eq. (13), restated here for convenience],

$$
\rho_{m}^{(d)}=\int d^{2} \beta \frac{e^{-|\beta|^{2} / n^{\prime}}}{\pi n^{\prime}} \mathcal{D}[\beta] \rho_{m} \mathcal{D}^{\dagger}[\beta] .
$$

In this case,

$$
\begin{aligned}
p_{+}(|\alpha|, \phi)= & \operatorname{Tr}_{m}\left[\Upsilon_{+}^{\dagger} \Upsilon_{+} \rho_{m}^{(\mathrm{d})}\right] \\
= & \int d^{2} \beta \frac{e^{-|\beta|^{2} / n^{\prime}}}{\pi n^{\prime}} \operatorname{Tr}_{m}\left[\mathcal{D}^{\dagger}[\beta] \Upsilon_{+}^{\dagger} \Upsilon_{+} \mathcal{D}[\beta] \rho_{m}\right] \\
= & \int d x^{\prime} d \beta_{r} \frac{e^{-\beta_{r}^{2} / n^{\prime}}}{\sqrt{\pi n^{\prime}}} P\left(x^{\prime}\right) \\
& \times \cos ^{2}\left(|\alpha|\left(x^{\prime}+2 \beta_{r}\right)+\frac{\phi}{2}\right) \\
= & \frac{1}{2}+\frac{e^{-4 n^{\prime}|\alpha|^{2}} \int d x P(x) \cos (2 x|\alpha|)}{2} \cos (\phi),
\end{aligned}
$$

where $\operatorname{Tr}_{m}$ denotes a trace over the mechanical degrees of freedom. We therefore see that any loss of coherence between the second and third grating reduces the amplitude of the oscillations in $p_{+}$by a factor $e^{-4 n^{\prime}\left|\alpha^{2}\right|}$.

\section{APPENDIX D: CLASSICAL INTERFERENCE PATTERNS}

To verify that oscillations are in fact due to quantum interference, we consider what types of interference patterns can be understood using a classical description of the resonator. Consider a classical (superscript cl) checkerboard phase-space probability density of dimensionless variables $(x, p)$,

$P^{\mathrm{cl}}(x, p)=\frac{1}{N} \exp \left[-\frac{x^{2}+p^{2}}{2 \sigma^{2}}\right] \cos ^{2}(\alpha x) \cos ^{2}(\alpha p)$,

where $N$ is a normalization factor and $\sigma$ and $\alpha$ are the width and wave number of the checkerboard pattern, respectively. This is the conditional state after the resonator has passed the first two gratings (of $\alpha=\alpha_{1}=\alpha_{2}$ ) separated by a quarter-period rotation. As the distribution rotates in phase space, we are interested in the wave-number components present in the reduced probability of $P^{\mathrm{cl}}(x)$, where

$P^{\mathrm{cl}}(x)=\int P^{\mathrm{cl}}(x \cos \theta+p \sin \theta, p \cos \theta-x \sin \theta) d p$

and $\theta$ is the phase-space rotation angle. If this probability distribution is now probed by a third grating, then the probability of finding the qubit in the $|+\rangle$ state is [from Eq. (12)]

$$
\begin{aligned}
p_{+}^{\mathrm{cl}}\left(\alpha_{3}, \phi\right)= & \int d x^{\prime} \cos ^{2}\left(\left|\alpha_{3}\right| x^{\prime}+\frac{\phi}{2}\right) P^{\mathrm{cl}}\left(x^{\prime}\right) \\
\propto & e^{-2 \sigma^{2}\left(\left|\alpha_{3}\right|^{2}+2\left|\alpha_{3}\right||\alpha|(\sin (\theta)+\cos (\theta))+2|\alpha|^{2}\right)} \\
& \times\left[e^{8\left|\alpha_{3}\right||\alpha| \sigma^{2} \cos (\theta)}+2 e^{2|\alpha| \sigma^{2}\left(2\left|\alpha_{3}\right| \cos (\theta)+|\alpha|\right)}+1\right] \\
& \times\left[e^{8\left|\alpha_{3}\right||\alpha| \sigma^{2} \sin (\theta)}+2 e^{2|\alpha| \sigma^{2}\left(2\left|\alpha_{3}\right| \sin (\theta)+|\alpha|\right)}+1\right] \\
& \times \cos (\phi),
\end{aligned}
$$

the amplitude of which peaks at $\left\{\Omega \tau_{2},\left|\alpha_{3} / \alpha\right|\right\}=\left\{\frac{1}{2} n \pi, 1\right\}$ and $\left\{\left(\frac{1}{2} n+\frac{1}{4}\right) \pi, \sqrt{2}\right\}$ for $\sigma \geq|\alpha|$. A plot of this function (Fig. 7) looks qualitatively the same as Fig. 6(b), with the difference being attributable to the superposition of

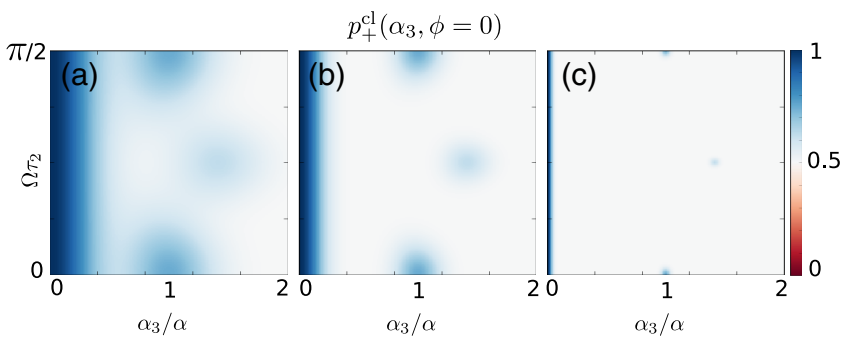

FIG. 7. Probability $p_{+}$of finding the qubit in the excited state if the resonator were described by a classical probability distribution using width $\sigma=0.5,1,5$ from (a) to (c). 
TABLE I. Parameters for a selection of implemented or proposed qubit-resonator coupled systems for carrying out the experiment in the main text. The systems are as follows: (1) this proposed device, (2) proposed levitated NV center in a magnetic field gradient, (3) implemented magnetic cantilever coupled to a NV center, (4) proposed optimization of the device in (3), (5) implemented NV center strain-coupled to diamond cantilever, (6) proposed combination of the Al beam from Ref. [75] with the qubit of Ref. [37], in a field of $10 \mathrm{mT}$, (7) proposed combination of the SiN membrane from Ref. [76] with the qubit of Ref. [37], in a field of $10 \mathrm{mT}$. The membrane is taken as supporting one arm of the qubit with length $l=300 \mu \mathrm{m}$. Column (8) is the proposed combination of the optimized cantilever from Ref. [55] with the qubit of Ref. [37]. The field from the cantilever is taken as the coupling to an enclosed area of $0.1 \mu \mathrm{m}^{-2}$. None of these systems enters the bare ultrastrong coupling regime where $\lambda / \Omega>1$. However, it is possible to enter the toggled ultrastrong coupling regime where $\lambda$ exceeds both the qubit and mechanical dephasing rates.

\begin{tabular}{|c|c|c|c|c|c|c|c|c|}
\hline $\begin{array}{l}\text { System } \\
\text { Reference }\end{array}$ & $\begin{array}{c}1 \\
\text { This work }\end{array}$ & $\begin{array}{c}2 \\
{[14]^{\dagger}}\end{array}$ & $\begin{array}{c}3 \\
{[55]^{\dagger}}\end{array}$ & $\begin{array}{c}4 \\
{[55]}\end{array}$ & $\begin{array}{c}5 \\
{[77]}\end{array}$ & $\begin{array}{c}6 \\
{[37,75]}\end{array}$ & $\begin{array}{c}7 \\
{[37,55]}\end{array}$ & $\begin{array}{c}8 \\
{[37,76]}\end{array}$ \\
\hline \multicolumn{9}{|c|}{ Parameters } \\
\hline Frequency $\Omega / 2 \pi(\mathrm{Hz})$ & $1.25 \times 10^{8}$ & $10^{5}$ & $8.0 \times 10^{4}$ & $10^{6}$ & $9.19 \times 10^{5}$ & $1.0 \times 10^{6}$ & $1.23 \times 10^{5}$ & $10^{6}$ \\
\hline Mass $m$ (amu) & $3 \times 10^{6}$ & $8 \times 10^{9}$ & $7 \times 10^{15}$ & $7 \times 10^{15}$ & $3 \times 10^{10}$ & $7 \times 10^{12}$ & $1 \times 10^{17}$ & $7 \times 10^{15}$ \\
\hline Quality factor $Q_{m}$ & $10^{5}$ & $\cdots$ & $2 \times 10^{2}$ & $10^{6}$ & $1 \times 10^{4}$ & $6 \times 10^{5}$ & $4 \times 10^{7}$ & $10^{6}$ \\
\hline Temperature $T(\mathrm{~K})$ & $3.3 \times 10^{-2}$ & $1 \times 10^{-3}$ & $3 \times 10^{2}$ & $1 \times 10^{-1}$ & $3 \times 10^{2}$ & $1.3 \times 10^{-1}$ & $1.8 \times 10^{-1}$ & $1 \times 10^{-1}$ \\
\hline Qubit $T_{2}^{*}$ & $2 \times 10^{-6}$ & $10^{-4}$ & $1.6 \times 10^{-4}$ & $2 \times 10^{-3}$ & $2 \times 10^{-6}$ & $2 \times 10^{-6}$ & $2 \times 10^{-6}$ & $2 \times 10^{-6}$ \\
\hline Coupling constant $\lambda / 2 \pi(\mathrm{Hz})$ & $4.2 \times 10^{6}$ & $1.5 \times 10^{3}$ & $1.6 \times 10^{1}$ & $2 \times 10^{4}$ & 1.8 & $2 \times 10^{5}$ & $9 \times 10^{3}$ & 2.4 \\
\hline \multicolumn{9}{|c|}{ Dimensionless figures of merit } \\
\hline Coupling parameter $\lambda / \Omega$ & $3 \times 10^{-2}$ & $2 \times 10^{-2}$ & $2 \times 10^{-4}$ & $2 \times 10^{-2}$ & $2 \times 10^{-6}$ & $2 \times 10^{-1}$ & $7 \times 10^{-2}$ & $2 \times 10^{-6}$ \\
\hline Mechanical parameter $\lambda / \kappa_{\mathrm{th}}$ & $6 \times 10^{2}$ & $\cdots$ & $5 \times 10^{-10}$ & $1 \times 10^{1}$ & $3 \times 10^{-9}$ & $5 \times 10^{1}$ & $8 \times 10^{1}$ & $1 \times 10^{-3}$ \\
\hline Qubit parameter $T_{2}^{*} \lambda / 2 \pi$ & 8 & $2 \times 10^{1}$ & $3 \times 10^{-3}$ & $4 \times 10^{1}$ & $4 \times 10^{-6}$ & $4 \times 10^{-1}$ & $2 \times 10^{-2}$ & $5 \times 10^{-6}$ \\
\hline
\end{tabular}

$\bar{~}{ }^{\dagger}$ In these references, the dephasing time $T_{2}^{*}$ is not directly reported, and we have therefore optimistically used the decoherence time $T_{2}$ instead.

momentum kicks that accompanies the measurement when the resonator is quantized. This confirms that the probability of finding the resonator in the $|+\rangle$ state can therefore be used to distinguish quantum interference patterns [Figs. 6(a) and 6(c)] from classical Moiré patterns that arise from a classical probability distribution (Fig. 7).

\section{APPENDIX E: OTHER DEVICE IMPLEMENTATIONS}

To assess the experimental feasibility of our scheme, Table I presents parameters of the resonator, the qubit, and the coupling strength for various devices that could be used to implement it. The challenge is to achieve ultrastrong coupling between the qubit and resonator without introducing either rapid dephasing of the qubit or thermal decoherence of the resonator. Assuming a toggled coupling, this requires that the coupling constant $\lambda / 2 \pi$ exceeds both the qubit dephasing rate $1 / T_{2}^{*}$ and the resonator thermal dephasing rate $\kappa_{\mathrm{th}}$, as tabulated in the last two rows of Table I. No existing device achieves this, although an optimized magnetic cantilever coupled to a NV center in diamond would be promising. Thus, the device of Fig. 1 is particularly attractive for investigating mesoscopic quantum interference in nanomechanics.

[1] K. Hornberger, S. Gerlich, P. Haslinger, S. Nimmrichter, and M. Arndt, Colloquium: Quantum Interference of Clusters and Molecules, Rev. Mod. Phys. 84, 157 (2012).
[2] S. Eibenberger, S. Gerlich, M Arndt, M. Mayor, and J. Tüxen, Matter-Wave Interference of Particles Selected from a Molecular Library with Masses Exceeding 10,000 amu, Phys. Chem. Chem. Phys. 15, 14696 (2013).

[3] L. Diósi, Models for Universal Reduction of Macroscopic Quantum Fluctuations, Phys. Rev. A 40, 1165 (1989).

[4] R. Penrose, On Gravity's Role in Quantum State Reduction, Gen. Relativ. Gravit. 28, 581 (1996).

[5] M. P. Blencowe, Effective Field Theory Approach to Gravitationally Induced Decoherence, Phys. Rev. Lett. 111, 021302 (2013).

[6] D. Kafri, J. M. Taylor, and G. J. Milburn, A Classical Channel Model for Gravitational Decoherence, New J. Phys. 16, 065020 (2014).

[7] I. Pikovski, M. Zych, F. Costa, and C. Brukner, Universal Decoherence Due to Gravitational Time Dilation, Nat. Phys. 11, 668 (2015).

[8] A. Bassi, K. Lochan, S. Satin, T. P. Singh, and H. Ulbricht, Models of Wave-Function Collapse, Underlying Theories, and Experimental Tests, Rev. Mod. Phys. 85, 471 (2013).

[9] W. Marshall, C. Simon, R. Penrose, and D. Bouwmeester, Towards Quantum Superpositions of a Mirror, Phys. Rev. Lett. 91, 130401 (2003).

[10] O. Romero-Isart, A. C. Pflanzer, F. Blaser, R. Kaltenbaek, N. Kiesel, M. Aspelmeyer, and J. I. Cirac, Large Quantum Superpositions and Interference of Massive Nanometer-Sized Objects, Phys. Rev. Lett. 107, 020405 (2011).

[11] J. Bateman, S. Nimmrichter, K. Hornberger, and $\mathrm{H}$. Ulbricht, Near-Field Interferometry of a Free-Falling Nanoparticle from a Point-like Source, Nat. Commun. 5, 4788 (2014). 
[12] M. Aspelmeyer, T. J. Kippenberg, and F. Marquardt, Cavity Optomechanics, Rev. Mod. Phys. 86, 1391 (2014).

[13] Z. Q. Yin, T. Li, X. Zhang, and L. M. Duan, Large Quantum Superpositions of a Levitated Nanodiamond through SpinOptomechanical Coupling, Phys. Rev. A 88, 033614 (2013).

[14] M. Scala, M. S. Kim, G. W. Morley, P. F. Barker, and S. Bose, Matter-Wave Interferometry of a Levitated Thermal Nano-Oscillator Induced and Probed by a Spin, Phys. Rev. Lett. 111, 180403 (2013).

[15] A. O'Connell, M. Hofheinz, M. Ansmann, R. Bialczak, M. Lenander, E. Lucero, M. Neeley, D. Sank, H. Wang, M. Weides, J. Wenner, J. M. Martinis, and A.N. Cleland, Quantum Ground State and Single-Phonon Control of a Mechanical Resonator, Nature (London) 464, 697 (2010).

[16] J. Chan, T.P. Mayer Alegre, A. H. Safavi-Naeini, J. T. Hill, A. Krause, S. Gröblacher, M. Aspelmeyer, and O. Painter, Laser Cooling of a Nanomechanical Oscillator into Its Quantum Ground State, Nature (London) 478, 89 (2011).

[17] J. D. Teufel, T. Donner, D. Li, J. Harlow, M. S. Allman, K. Cicak, A. J. Sirois, J. D. Whittaker, K. W. Lehnert, and R. W. Simmonds, Sideband Cooling of Micromechanical Motion to the Quantum Ground State, Nature (London) 475, 359 (2011).

[18] E. E. Wollman, C. U. Lei, A. J. Weinstein, J. Suh, A. Kronwald, F. Marquardt, A. A. Clerk, and K. C. Schwab, Quantum Squeezing of Motion in a Mechanical Resonator, Science 349, 952 (2015).

[19] F. Lecocq, J. B. Clark, R. W. Simmonds, J. Aumentado, and J. D. Teufel, Quantum Nondemolition Measurement of a Nonclassical State of a Massive Object, Phys. Rev. X 5, 041037 (2015).

[20] J.-M. Pirkkalainen, E. Damskägg, M. S. Brandt, F. Massel, and M. A. Sillanpää, Squeezing of Quantum Noise of Motion in a Micromechanical Resonator, Phys. Rev. Lett. 115, 243601 (2015).

[21] J.-M. Pirkkalainen, S. U. Cho, J. Li, G. S. Paraoanu, P. J. Hakonen, and M. A. Sillanpää, Hybrid Circuit Cavity Quantum Electrodynamics with a Micromechanical Resonator, Nature (London) 494, 211 (2013).

[22] F. Lecocq, J. D. Teufel, J. Aumentado, and R. W. Simmonds, Resolving the Vacuum Fluctuations of an Optomechanical System using an Artificial Atom, Nat. Phys. 11, 635 (2015).

[23] A. D. Armour, M. P. Blencowe, and K. C. Schwab, Entanglement and Decoherence of a Micromechanical Resonator via Coupling to a Cooper-Pair Box, Phys. Rev. Lett. 88, 148301 (2002).

[24] A. D. Armour and M. P. Blencowe, Probing the Quantum Coherence of a Nanomechanical Resonator Using a Superconducting Qubit: I. Echo Scheme, New J. Phys. 10, 095004 (2008).

[25] M. P. Blencowe and A. D. Armour, Probing the Quantum Coherence of a Nanomechanical Resonator Using a Superconducting Qubit: II. Implementation, New J. Phys. 10, 095005 (2008).

[26] M. Wallquist, K. Hammerer, P. Rabl, M. Lukin, and P. Zoller, Hybrid Quantum Devices and Quantum Engineering, Phys. Scr. T137, 014001 (2009).
[27] N. Didier, S. Pugnetti, Y. M. Blanter, and R. Fazio, Quantum Transducer in Circuit Optomechanics, Solid State Commun. 198, 61 (2014).

[28] J. Chaste, A. Eichler, J. Moser, G. Ceballos, R. Rurali, and A. Bachtold, A Nanomechanical Mass Sensor with Yoctogram Resolution, Nat. Nanotechnol. 7, 301 (2012).

[29] J. Moser, J. Güttinger, A. Eichler, M. J. Esplandiu, D. E. Liu, M. I. Dykman, and A. Bachtold, Ultrasensitive Force Detection with a Nanotube Mechanical Resonator, Nat. Nanotechnol. 8, 493 (2013).

[30] M. Ringbauer, T. J. Weinhold, L. A Howard, A. G. White, and M. R. Vanner, Generation of Mechanical Interference Fringes by Multi-Photon Counting, New J. Phys. 20, 053042 (2018).

[31] M. Arndt and K. Hornberger, Testing the Limits of Quantum Mechanical Superpositions, Nat. Phys. 10, 271 (2014).

[32] A. Pályi, P. R. Struck, M. S. Rudner, K. Flensberg, and G. Burkard, Spin-Orbit-Induced Strong Coupling of a Single Spin to a Nanomechanical Resonator, Phys. Rev. Lett. 108, 206811 (2012).

[33] C. Ohm, C. Stampfer, J. Splettstoesser, and M. Wegewijs, Readout of Carbon Nanotube Vibrations Based on SpinPhonon Coupling, Appl. Phys. Lett. 100, 143103 (2012).

[34] T. Pei, A. Pályi, M. Mergenthaler, N. Ares, A. Mavalankar, J. H. Warner, G. A. D. Briggs, and E. A. Laird, Hyperfine and Spin-Orbit Coupling Effects on Decay of Spin-Valley States in a Carbon Nanotube, Phys. Rev. Lett. 118, 177701 (2017).

[35] T. A. Palomaki, J. D. Teufel, R. W. Simmonds, and K. W. Lehnert, Entangling Mechanical Motion with Microwave Fields, Science 342, 710 (2013).

[36] P. Treutlein, C. Genes, K. Hammerer, M. Poggio, and P. Rabl, Hybrid Mechanical Systems, in Cavity Optomechanics: Nano- and Micromechanical Resonators Interacting with Light (Springer, New York, 2014), p. 327, https:// doi.org/10.1007/978-3-642-55312-7_14.

[37] J. Braumüller, M. Sandberg, M. R. Vissers, A. Schneider, S. Schlör, L. Grünhaupt, H. Rotzinger, M. Marthaler, A. Lukashenko, A. Dieter, A. V. Ustinov, M. Weides, and D. P. Pappas, Concentric Transmon Qubit Featuring Fast Tunability and an Anisotropic Magnetic Dipole Moment, Appl. Phys. Lett. 108, 032601 (2016).

[38] V. Sazonova, Y. Yaish, H. Ustunel, D. Roundy, T. A. Arias, and P. L. McEuen, A Tunable Carbon Nanotube Electromechanical Oscillator, Nature (London) 431, 284 (2004).

[39] E. A. Laird, F. Pei, W. Tang, G. A. Steele, and L. Kouwenhoven, A High Quality Factor Carbon Nanotube Mechanical Resonator at $39 \mathrm{GHz}$, Nano Lett. 12, 193 (2012).

[40] A. K. Hüttel, G. A. Steele, B. Witkamp, M. Poot, L. P. Kouwenhoven, and H.S. J. van der Zant, Carbon Nanotubes as Ultrahigh Quality Factor Mechanical Resonators, Nano Lett. 9, 2547 (2009).

[41] P. Jarillo-Herrero, J. A. Dam, and L. P. Kouwenhoven, Quantum Supercurrent Transistors in Carbon Nanotubes, Nature (London) 439, 953 (2006).

[42] J. Cleuziou, W. Wernsdorfer, V. Bouchiat, T. Ondarcuhu, and M. Monthioux, Carbon Nanotube Superconducting Quantum Interference Device, Nat. Nanotechnol. 1, 53 (2006). 
[43] B. H. Schneider, S. Etaki, H. S. J. van der Zant, and G. A. Steele, Coupling Carbon Nanotube Mechanics to a Superconducting Circuit, Sci. Rep. 2, 599 (2012).

[44] J. Moser, A. Eichler, J. Güttinger, M. I. Dykman, and A. Bachtold, Nanotube Mechanical Resonators with Quality Factors of up to 5 Million, Nat. Nanotechnol. 9, 1007 (2014).

[45] F. Xue, Y. D. Wang, C. P. Sun, H. Okamoto, H. Yamaguchi, and K. Semba, Controllable Coupling between Flux Qubit and Nanomechanical Resonator by Magnetic Field, New J. Phys. 9, 35 (2007).

[46] S. Etaki, M. Poot, I. Mahboob, K. Onomitsu, H. Yamaguchi, and H. S. J. van der Zant, Motion Detection of a Micromechanical Resonator Embedded in a D. C. SQUID, Nat. Phys. 4, 785 (2008).

[47] O. Shevchuk, G. A. Steele, and Y. M. Blanter, Strong and Tunable Couplings in Flux-Mediated Optomechanics, Phys. Rev. B 96, 014508 (2017).

[48] H. Üstünel, D. Roundy, and T. A. Arias, Modeling a Suspended Nanotube Oscillator, Nano Lett. 5, 523 (2005).

[49] B. Witkamp, M. Poot, and H. S. J. van der Zant, BendingMode Vibration of a Suspended Nanotube Resonator, Nano Lett. 6, 2904 (2006).

[50] N. Ares, T. Pei, A. Mavalankar, M. Mergenthaler, J. H. Warner, G. A. D. Briggs, and E. A. Laird, Resonant Optomechanics with a Vibrating Carbon Nanotube and a RadioFrequency Cavity, Phys. Rev. Lett. 117, 170801 (2016).

[51] M. Poot and H. S. J. van der Zant, Mechanical Systems in the Quantum Regime, Phys. Rep. 511, 273 (2012).

[52] J. Koch, T. M. Yu, J. M. Gambetta, A. A. Houck, D. I. Schuster, J. Majer, A. Blais, M. H. Devoret, S. M. Girvin, and R.J. Schoelkopf, Charge-Insensitive Qubit Design Derived from the Cooper Pair Box, Phys. Rev. A 76, 042319 (2007).

[53] F. Mallet, F. R. Ong, A. Palacios-Laloy, F. Nguyen, P. Bertet, D. Vion, and D. Esteve, Single-Shot Qubit Readout in Circuit Quantum Electrodynamics, Nat. Phys. 5, 791 (2009).

[54] L. DiCarlo, J. M. Chow, J. M. Gambetta, L. S. Bishop, B. R. Johnson, D. I. Schuster, J. Majer, A. Blais, L. Frunzio, S. M. Girvin, and R. J. Schoelkopf, Demonstration of Two-Qubit Algorithms with a Superconducting Quantum Processor, Nature (London) 460, 240 (2009).

[55] S. Kolkowitz, A. C. B. Jayich, Q. P. Unterreithmeier, S. D. Bennett, P. Rabl, J. G. E. Harris, and M. D. Lukin, Coherent Sensing of a Mechanical Resonator with a Single-Spin Qubit, Science 335, 1603 (2012).

[56] D. I. Bradley, R. E. George, D. Gunnarsson, R. P. Haley, H. Heikkinen, Yu. A. Pashkin, J. Penttilä, J. R. Prance, M. Prunnila, L. Roschier, and M. Sarsby, Nanoelectronic Primary Thermometry below $4 \mathrm{mK}$, Nat. Commun. 7, 10455 (2016).

[57] M. R. Vanner, I. Pikovski, G. D. Cole, M. S. Kim, C. Brukner, K. Hammerer, G. J. Milburn, and M. Aspelmeyer, Pulsed Quantum Optomechanics, Proc. Natl. Acad. Sci. U.S.A. 108, 16182 (2011).

[58] S. D. Bennett, S. Kolkowitz, Q. P. Unterreithmeier, P. Rabl, A. C. Bleszynski Jayich, J. G. E. Harris, and M. D. Lukin, Measuring Mechanical Motion with a Single Spin, New J. Phys. 14, 125004 (2012).
[59] A. Asadian, C. Brukner, and P. Rabl, Probing Macroscopic Realism via Ramsey Correlation Measurements, Phys. Rev. Lett. 112, 190402 (2014).

[60] If $\alpha$ is real, Eq. (6) leads to a position basis superposition, which is a $\pi / 2$ rotation of the momentum superposition. In this way, any complex $\alpha$ can be understood in terms of a purely imaginary $\alpha$, followed by a rotation. Without loss of generality, and to keep the analogy to optical standing-wave gratings, we therefore consider $\operatorname{Re}(\alpha)=0$.

[61] Equation (9) can be intuitively understood from Eq. (8) by noting that for $\operatorname{Re}(\alpha=0), \mathcal{D}(\alpha)=e^{i|\alpha|\left(a+a^{\dagger}\right)}$ and by decomposing the exponentials in Eq. (8) into trigonometric functions.

[62] W. H. Zurek, Sub-Planck Structure in Phase Space and Its Relevance for Quantum Decoherence, Nature (London) 412, 712 (2001).

[63] F. Toscano, D. A. R. Dalvit, L. Davidovich, and W. H. Zurek, Sub-Planck Phase-Space Structures and Heisenberg-Limited Measurements, Phys. Rev. A 73, 023803 (2006).

[64] Z. Leghtas, G. Kirchmair, B. Vlastakis, R. J. Schoelkopf, M. H. Devoret, and M. Mirrahimi, Hardware-Efficient Autonomous Quantum Memory Protection, Phys. Rev. Lett. 111, 120501 (2013).

[65] S. Nimmrichter and K. Hornberger, Macroscopicity of Mechanical Quantum Superposition States, Phys. Rev. Lett. 110, 160403 (2013).

[66] S. Gerlich, S. Eibenberger, M. Tomandl, S. Nimmrichter, K. Hornberger, P. J. Fagan, J. Tüxen, M. Mayor, and M. Arndt, Quantum Interference of Large Organic Molecules, Nat. Commun. 2, 263 (2011).

[67] S. Dimopoulos, P. W. Graham, J. M. Hogan, M. A. Kasevich, and S. Rajendran, Gravitational Wave Detection with Atom Interferometry, Phys. Lett. B 678, 37 (2009).

[68] S. Nimmrichter, K. Hornberger, P. Haslinger, and M. Arndt, Testing Spontaneous Localization Theories with Matter-Wave Interferometry, Phys. Rev. A 83, 043621 (2011).

[69] The macroscopicities quoted here are obtained from Refs. [31,65].

[70] R. Manenti, A. F. Kockum, A. Patterson, T. Behrle, J. Rahamim, G. Tancredi, F. Nori, and P. J. Leek, Circuit Quantum Acoustodynamics with Surface Acoustic Waves, Nat. Commun. 8, 975 (2017).

[71] Y. Chu, P. Kharel, W. H. Renninger, L. D. Burkhart, L. Frunzio, P. T. Rakich, and R. J. Schoelkopf, Quantum Acoustics with Superconducting Qubits, Science 358, 199 (2017).

[72] C. Wan, M. Scala, G. W. Morley, A. T. M. A. Rahman, H. Ulbricht, J. Bateman, P. F. Barker, S. Bose, and M. S. Kim, Free Nano-Object Ramsey Interferometry for Large Quantum Superpositions, Phys. Rev. Lett. 117, 143003 (2016).

[73] S. G. Hofer, K. W. Lehnert, and K. Hammerer, Proposal to Test Bell's Inequality in Electromechanics, Phys. Rev. Lett. 116, 070406 (2016).

[74] J. A. Schreier, A. A. Houck, J. Koch, D. I. Schuster, B. R. Johnson, J. M. Chow, J. M. Gambetta, J. Majer, L. Frunzio, M. H. Devoret, S. M. Girvin, and R. J. Schoelkopf, Suppressing Charge Noise Decoherence in Superconducting Charge Qubits, Phys. Rev. B 77, 180502 (2008). 
[75] J. D. Teufel, T. Donner, M. Castellanos-Beltran, J. Harlow, and K. W. Lehnert, Nanomechanical Motion Measured with an Imprecision below that at the Standard Quantum Limit, Nat. Nanotechnol. 4, 820 (2009).

[76] M. Yuan, V. Singh, Y. M. Blanter, and G. A. Steele, Large Cooperativity and Microkelvin Cooling with a
Three-Dimensional Optomechanical Cavity, Nat. Commun. 6, 8491 (2015).

[77] S. Meesala, Y. I. Sohn, H. A. Atikian, S. Kim, M. J. Burek, J. T. Choy, and M. Loncar, Enhanced Strain Coupling of Nitrogen-Vacancy Spins to Nanoscale Diamond Cantilevers, Phys. Rev. Applied 5, 034010 (2016). 